\title{
Metastatic EML4-ALK fusion detected by circulating DNA genotyping in an EGFR- mutated NSCLC patient and successful management by adding ALK inhibitors: a case report
}

\author{
Wenhua Liang ${ }^{1,2+}$, Qihua He $e^{1,2+}$, Ying Chen ${ }^{1,2+}$, Shaokun Chuai ${ }^{3}$, Weiqiang Yin ${ }^{1,2}$, Wei Wang ${ }^{1,2}$, Guilin Peng ${ }^{1,2}$, \\ Caicun Zhou ${ }^{1,2,4^{*}}$ and Jianxing $\mathrm{He}^{1,2^{*}}$
}

\begin{abstract}
Background: Rebiopsy is highly recommended to identify the mechanism of acquired resistance to EGFR-TKIs in advanced lung cancer. Recent advances in multiplex genotyping based on circulating tumor DNA (ctDNA) provide a strong and non-invasive alternative for detection of the resistance mechanism.

Case presentation: Here we report a multiple metastatic NSCLC patient who was detected to have pure EGFR 19 exon deletion (negative for EML4-ALK and ROS1 in both IHC-based and sequencing assay) in the primary lesion and responded to first-line and second-line EGFR-TKI treatments (erlotinib then HY-15772). At 8 months, most lesions remained well controlled except for the liver metastases which presented dramatic progression. Considering the high risk of bleeding in rebiopsy of hepatic lesions, we conducted a multiplex genomic profiling with ctDNA. Results reported coexistence of EGFR mutation and EML4-ALK gene translocation in plasma which heavily indicated that ALK was the primary reason for progression of the liver lesions. This deduction was supported by the repeated response to ALK inhibitors (crizotinib then AP26113) of the hepatic metastases.

Conclusions: This is the first report of the existence of ALK rearrangement in metastatic lesions in an EGFR mutated patient. It highlighted the feasibility and advantages of using ctDNA multiplex genotyping in identifying the heterogeneity across lesions and the resistance mechanism of targeted treatments.
\end{abstract}

Keywords: NSCLC, EGFR mutation, EML4-ALK rearrangement, Co-existence

\section{Background}

Advances in geno-typing have changed the clinical practice of treatment of non-small cell lung cancer (NSCLC), especially non-squamous types where driver mutations, e.g. epidermal growth factor receptor (EGFR) mutations

\footnotetext{
*Correspondence: caicunzhoudr@163.com; drijanxing.he@gmail.com Co-first authors are Wenhua Liang, Qihua He and Ying Chen

${ }^{\dagger}$ Equal contributors

${ }^{1}$ Department of Thoracic Surgery and Oncology, The First Affiliated Hospital of Guangzhou Medical University, Guangzhou, China

${ }^{2}$ Guangzhou Institute of Respiratory Disease \& China State Key Laboratory of Respiratory Disease \& National Clinical Research Center for Respiratory Disease, No. 151, Yanjiang Rd, Guangzhou 510120, Guangdong Province, PR China

Full list of author information is available at the end of the article
}

and echinoderm microtubule-associated protein-like 4anaplastic lymphoma kinase (EML4-ALK) translocation are commonly present. Agents that target EGFR activating mutations (gefitinib, erlotinib, and afatinib, etc.) or ALK rearrangement (crizotinib, etc.) derive significantly greater benefits than cytotoxic chemotherapy in patients who harbor these gene alterations, which is consistently proved by extensive large-scale randomized controlled trials $[1,2]$. In order to deliver an appropriate first-line treatment regimen, detection of EGFR mutation and ALK rearrangement are recommended as routine genetic profiling for non-squamous NSCLC or non-smoking populations [3]. In recent years, some selective inhibitors 
that can overcome the resistance to first generation inhibitors of these driver alterations have also been developed, e.g., AZD9291 and CO-1686, some more effective inhibitors against both EGFR sensitizing and resistance T790M mutations [4]; or ceretinib, aletinib and AP26113, the agents that are effective for both ALK fusion and some secondary gatekeeper mutations [5].

Direct sequencing and amplification refractory mutation system (ARMS)-PCR are the common testing methods for EGFR mutations. FISH, RT-PCR and Ventana IHC are all currently accepted methods for detection of ALK rearrangement. In recent years, the development and advances in high throughput next-generation sequencing (NGS) have allowed the simultaneous profiling of alterations in multiple genes [6]. Circulating tumor DNA (ctDNA) is released or excreted by tumour cells and circulates in the blood of a cancer patient; analysis of the fraction of mutant-alleles from ctDNA compared to normal-alleles from the patients' normal genome provides opportunities for minimally-invasive cancer diagnosis and tumor monitoring [7]. Detection with ctDNA, which originates from all potential lesions, could overcome the disadvantages of single site biopsy given that the intratumoral and inter-lesional heterogeneity is common $[6,8]$. Enrichment of plasma ctDNA and incorporation with the next generation deep sequencing techniques allow us to simultaneously detect the gene alterations of interest, e.g. EGFR/BRAF/HER2 mutations, ALK/ROS1/RET rearrangements, MET amplification, etc. in NSCLC, especially when it is difficult to obtain sufficient tissue samples.

EGFR mutations and ALK rearrangement are generally considered to be mutually exclusive. However, some recent surveys and case reports showed co-existence of the two alterations within the same lesion $[9,10]$. Here, we report an advanced NSCLC case with EGFR exon 19 deletion who experienced single-site progression in the liver after primary response to EGFR-TKI treatments and showed good response when adding crizotinib after the detection of ALK rearrangement signal through ctDNA. This special case highlights the feasibility and necessity of using ctDNA multiplex genomic profiling as an alternative approach in molecular diagnosis of NSCLC or in the exploration of the underlying mechanism in resistance to targeted therapies. Moreover, it encourages us to re-evaluate the heterogeneity across lesions of metastatic NSCLC.

\section{Case Presentation}

A 46-year-old woman with stage IVb lung adenocarcinoma of the left upper lobe and extensive metastases (mediastinal lymph nodes, bilateral lung, liver, brain, multiple vertebrae, pelvis, adrenal glands, retroperitoneal lymph nodes, etc.) was confirmed to harbor EGFR 19 exon deletion by ARMS-PCR. She was negative for
EML4-ALK and ROS1 by ventana IHC staining. Evaluation after 1 month and 3 months of erlotinib $150 \mathrm{mg}$ Qd treatment showed good partial response across all lesions. However, the patient presented with severe shortness of breath after 4 months. CT scan showed rapid progression of the distributed pulmonary and hepatic lesions. Based on the imaging features and clinical symptoms, it was initially difficult to differentiate whether the patient had interstitial pneumonia due to erlotinib, or lymphangitis carcinomatosa. Thus, we firstly withdrew erlotinib and delivered methylprednisolone pulse therapy (500 mg qd * 5 days). However, no improvement was observed, which lead us to the diagnosis of lymphangitis carcinomatosa due to disease progression. Pemetrexed $0.8 \mathrm{~g}$ plus bevacizumab $300 \mathrm{mg}$ (the tumor board decided to use a platinum-free regimen due to low tolerance to platinum of this patient, ECOG performance score 2-3), was then administered, but the lesions continued to grow rapidly. Noninvasive positive pressure ventilation was applied when the patient experienced type I respiratory failure. The patient took HY-15772, an active pharmaceutical ingredient (API) that was known for AZD9291, 100 mg qd on her own volition. She recovered from hyoxemia gradually and CT review showed tumor remission at 1 week after starting HY-15772 API. The patient continued to take HY-15772 API for 2 months. CT-scan showed that all lesions were controlled except for the liver metastases in the left lobe which underwent dramatic bulky progression. Multidisciplinary consultation suggested that a needle biopsy of the liver lesions was not preferable due to the high risk of procedure-related bleeding. Thus, we decided to obtain peripheral blood for ctDNA multiplex genotyping analysis using the capture probe baits sequencing platform (methods and gene list were provided in Additional file 1). Meanwhile, a dose of gemcitabine chemotherapy $1.4 \mathrm{~g}$ on day 1 and day 8 was administered. During the intermission, the ctDNA analysis reported the existence of both EGFR 19 exon L747S non-shifting deletion (abundance $48.5 \%$ ) and EML4-ALK rearrangement (abundance $10.09 \%$ ) in the plasma (No other druggable alteration was found especially in domains of MET). Upon the second cycle the gemcitabine chemotherapy, the patient complained of an obvious sensation of mass in the upper abdomen and the inability to keep food or drink down. We suggested the patient add crizotinib and continue HY-15772 API. After 5 days of additional crizotinib treatment, the patient was able to drink and eat and had the sensation of mass shrinkage. CT scan after 1 month reavealed significant remission of the lesions in the left lobe of the liver (plasma ALK abundance decreased to $2.79 \%$ ). After 2 months of crizotinib, liver metastases especially those in the left lobe again progressed, which was manifested as abdominal distention and edema of bilareal lower extremity 
(plasma ALK abundance increased to $14.59 \%$ ). The patient switched the ALK inhibitor to AP26113 API at a dose of $180 \mathrm{mg}$ Qd on her own volition and quickly regained good remission of the hepatic lesions (plasma ALK abundance decreased to $0.00 \%$ ). In addition, plasma ALK fusion was not detected. Figure 1

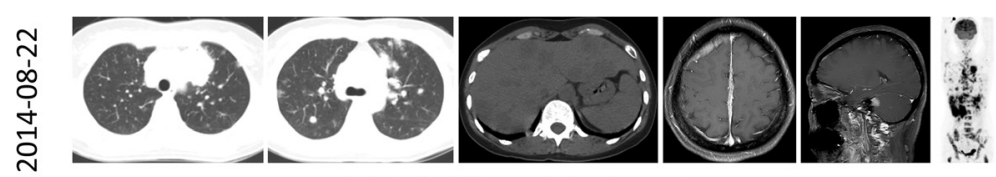

Erlotinib $150 \mathrm{mg}$ Qd $\times 3$ months

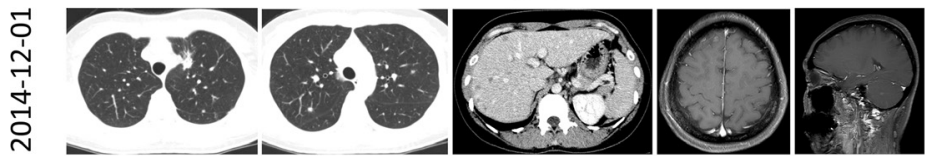

Erlotinib $150 \mathrm{mg}$ Qd $\times 1$ month

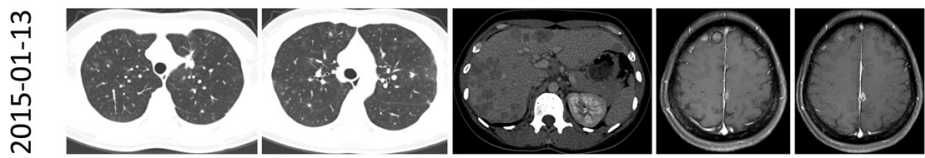

Pemetrexed $0.8 \mathrm{~g}$ plus Bevacizumab $300 \mathrm{mg} \times 1$ cycle

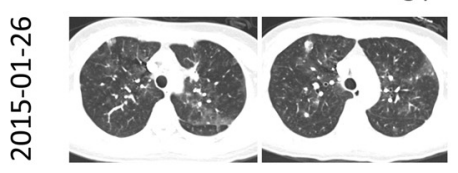

HY-15772 API 100 mg Qd $\times 1$ week

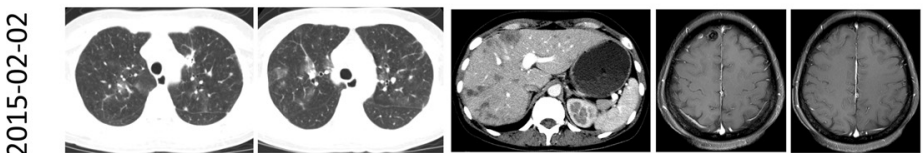

HY-15772 API 100 mg Qd × 2 months
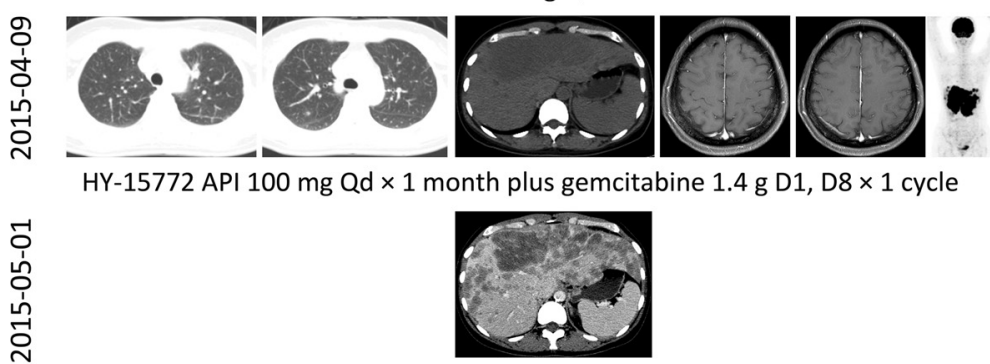

HY-15772 API $100 \mathrm{mg}$ Qd plus crizotinib $250 \mathrm{mg}$ Bid $\times 1$ month

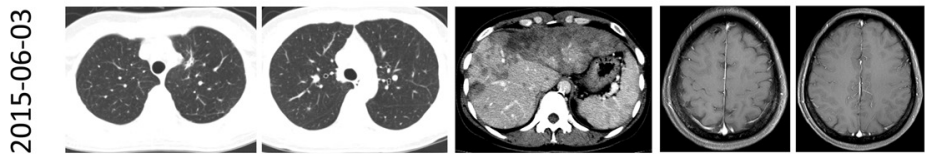

HY-15772 API $100 \mathrm{mg}$ Qd plus crizotinib $250 \mathrm{mg}$ Bid $\times 1$ month

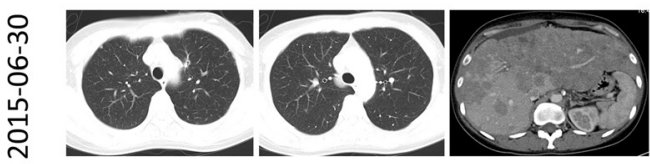

HY-15772 API 100 mg Qd plus AP26113 API 180mg Qd × 1 month

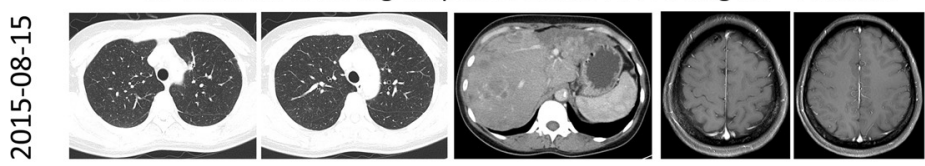

HY-15772 API 100 mg Qd plus AP26113 API 180mg Qd (continued)

Fig. 1 Changes in image during treatments. API, active pharmaceutical ingredient 
illustrated the change of the image presentation and treatments. Upon submission of this report, the patient is still receiving a combination treatment of HY-15772 and AP26113 API without any signs of disease worsening. Re-analysis of the biopsy tissue from the primary lesion through the same multiplex genomic platform showed pure EGFR exon 19 deletion without any signal for EML4-ALK fusion. In addition, repeated analyses indicated the presence of TP53 Y236D mutations in 7 exon with more than $50 \%$ abundance. Figure 1 illustrated the flow of treatments and image evaluation.

\section{Discussions}

The current recommendation for management of singlesite regional progression in resistance to EGFR-TKIs is to perform localized therapies and rebiopsy when available [11]. In this case, the progressed liver metastases were large, bulky, and scattered. Radiotherapy or radiofrequency ablation was not an appropriate option. Metastatic hepatic lesions were supplied by portal vein system rather than hepatic artery system. Thus, transcatheter arterial chemoembolization (TACE) might not derive significant benefits. On the other hand, this case progressed dramatically after showing resistance to EGFR-TKIs. In this case, switching to chemotherapy is suggested according to what IMPRESS trial indicated [12]. Considering the previous evidence of the patients' insensitivity to pemetrexed, we decided to use gemcitabine single agent chemotherapy. Meanwhile, we continued the use of HY-15772 API since all extra-hepatic lesions remained stable or even undetectable in the images, which suggested these lesions remained sensitive to HY-15772 API.

Rebiopsy was another issue worth clarifying. In this case, as the entire left lobe of the liver was occupied by metastatic lesions that were loosened in structure, multidisciplinary consultation opinions suggested that conducting a fine-needle biopsy would be dangerous due to the high risk of bleeding. Recent advances in non-invasive rebiopsy approaches such as multiplex genomic profiling of ctDNA provided us an alternative [13]. We decided to obtain peripheral blood from this patient to perform a NSCLCrelated multiplex genotyping, in order to clarify the resistance mechanism. Surprisingly, in addition to the 19del EGFR mutation that was previously discovered in the primary lesion, EML4-ALK fusion with relatively high abundance in plasma was detected. Given that the extrahepatic lesions were all under control, indicating their lasting response to HY-15772 API, we added Crizotinib, the only approved agent to inhibit ALK-driving tumors, to the regimen without withdrawing the EGFR suppressor. Previous reports suggested no significant superimposed toxicity in dual treatment with EGFR and ALK inhibitors [14]. In this case, the patient presented rapid relief without any grade III adverse events. Undoutedly, the timely addition of ALK inhibitors salvaged the patient as previous gemcitabine showed no efficacy.

Though some previous studies reported co-existence of EGFR and ALK in the same lesions, this is the first report showing ALK rearragement in distant metastatic lesions in a patient potentially harboring pure EGFR mutations in the primary lesion. Since rebiopsy for the liver metastases in the left lobe was not an option in this case, we had no direct evidence that the liver progression was driven by ALK fusion. However, the great abundance of EML4-ALK fusion signals in the plasma upon disease progression and the significant response after adding crizotinib (as well as the AP26113 API after resistance to crizotinib) strongly supported the existence of ALK fusion in the growing liver metastases. In addition, it was not likely that the primary lesion harbored concurrent EGFR mutation and ALK fusion because both routine ARMS and NGS re-analysis showed that no ALK fusion was detected and the pulmonary lesions were all in good control by HY-15772 API when the hepatic metastases progressed. Such heterogeneity might be more common than we have previously acknowledged, and might have a great impact on treatment strategy. In the past, we could only detect genetic alterations with site by site tissue samples. It was too difficult to obtain tissues from all lesions; multiplex genotyping with ctDNA might help address this issue. Thus, with the current technology, we should reevaluate the heterogeneity across different lesions and their incidence. Of course, the sensitivity of ctDNA examination and the substantial validation require further efforts.

We cannot determine whether the ALK fusion was inherent or if it was an evolvement after treatment. One possibility is the clonal selection mechanism, EML4-ALK fusion emerged when EGFR mutations were suppressed. However, in both the treatment with erlotinib and subsequent HY-15772 API, the hepatic lesions showed good response to these EGFR-TKIs, which suggested no or minimal ALK fusion at that point. But some reports have also revealed responsiveness to EGFR-TKIs in patients with ALK fusion or dual alterations [15]. Another potential reason is the inducement or de novo activation of EML4-ALK fusion after EGFR-TKI treatments since repeated analyses showed consistent high abundance (over $50 \%$ ) of TP53 mutation which heavily indicated to the presence of loss of heterozygosity (LOH) phenomena [16]. Genome instability might increase the risk for inducing other driver gene altrations. Further studies on the mechanism is warranted.

\section{Conclusions}

In conclusion, this is the first report of the existence of ALK rearrangement in metastatic lesions in an EGFR 
mutated patient. It highlighted the feasibility and advantages of using ctDNA multiplex genotyping in identifying the resistance mechanism of targeted treatments. Further well designed studies on its true effectiveness and criteria for elegible patient selection are warranted. In addition, ctDNA multiplex genotyping could benifit us in studying the heterogeneity across lesions of lung cancer.

\section{Availability of data and materials}

Any additional supporting data involving details of clinical and genetic analysis can be found in the electronic medical record system of the First Affiliated Hospital of Guangzhou Medical University and are available upon request from the corresponding author.

\section{Additional file}

Additional file 1: Platform and Gene-list for the Multiplex Genotyping of Circulating Tumor DNA. (DOC $45 \mathrm{~kb}$ )

\section{Abbreviation}

EGFR: Epidermal growth factor receptor; EML4-ALK: Echinoderm microtubuleassociated protein-like 4-anaplastic lymphoma kinase; IHC: Immunohistochemistry; TKl: Tyrosine kinase inhibitor.

\section{Competing interest}

All authors declare no conflict of interests.

\section{Authors' contribution}

$\mathrm{LWH}, \mathrm{HQH}, \mathrm{CY}, \mathrm{ZCC}$ and $\mathrm{HJX}$ desgined the study and collected all clinical data, participated in the interpretation of data and drafted the manuscript. CSK carried out the sequencing and bioinformatical analysis. YWQ, WW and PGL participated in acquisition and interpretation of data. All authors have read and approved the manuscript of this case report.

\section{Acknowledgement}

We thank Dan Zhou, Fang Luo, Haifang Zheng in Burning Rock Biotechnology Co. Ltd., Guangzhou, China for providing sequencing and bioinformatics support, and thank Lindsey Hamblin for language suggestions.

\section{Ethics}

This case report has been approved by the ethics committee of the First Affiliated Hospital of Guangzhou Medical University.

\section{Funding}

1. Guangdong Doctoral Launching Program, Grant No. 2014A030310460; 2. Doctoral Launching Program of Guangzhou Medical University, Grant No. 2014C27;

3. Guangzhou Health and Medical Collaborative Innovative Major Special Projects, Grant No. 201400000001-2.

\section{Informed consent}

Written informed consent was obtained from the patient for publication of this Case report and any accompanying images. A copy of the written consent is available for review by the Editor of this journal.

\section{Author details}

'Department of Thoracic Surgery and Oncology, The First Affiliated Hospital of Guangzhou Medical University, Guangzhou, China. ${ }^{2}$ Guangzhou Institute of Respiratory Disease \& China State Key Laboratory of Respiratory Disease \& National Clinical Research Center for Respiratory Disease, No. 151, Yanjiang Rd, Guangzhou 510120, Guangdong Province, PR China. ${ }^{3}$ Burning Rock Biotechnology Co. Ltd., Guangzhou, China. ${ }^{4}$ Department of Oncology,
Shanghai Pulmonary Hospital, Tongji University School of Medicine, Shanghai, China.

Received: 31 August 2015 Accepted: 27 January 2016

Published online: 05 February 2016

References

1. Lee CK, Brown C, Gralla RJ, Hirsh V, Thongprasert S, Tsai CM, et al. Impact of EGFR inhibitor in non-small cell lung cancer on progression-free and overall survival: a meta-analysis. J Natl Cancer Inst. 2013;105(9):595-605.

2. Qian H, Gao F, Wang H, Ma F. The efficacy and safety of crizotinib in the treatment of anaplastic lymphoma kinase-positive non-small cell lungcancer: a meta-analysis of clinical trials. BMC Cancer. 2014;14:683.

3. National Comprehensive Cancer Network. NCCN Clinical Practice Guidelines in OncologyTM. Non-Small Cell Lung Cancer. V5.2015. http://www.nccn.org/ professionals/physician_gls/PDF/nscl.pdf. Accessed March; 2015.

4. Gray J, Haura E. Update on third-generation EGFR tyrosine kinase inhibitors. Transl Lung Cancer Res. 2014;3(6):360-2.

5. Iragavarapu C, Mustafa M, Akinleye A, Furqan M, Mittal V, Cang S, et al. Novel ALK inhibitors in clinical use and development. J Hematol Oncol. 2015;8(1):17.

6. Li T, Kung HJ, Mack PC, Gandara DR. Genotyping and genomic profiling of non-small-cell lung cancer: implications for current and future therapies. J Clin Oncol. 2013;31(8):1039-49.

7. Uchida J, Kato K, Kukita Y, Kumagai T, Nishino K, Daga H, et al. Diagnostic Accuracy of Noninvasive Genotyping of EGFR in Lung Cancer Patients by Deep Sequencing of Plasma Cell-Free DNA. Clin Chem 2015. [Epub ahead of print].

8. O'Brien CP, Taylor SE, O'Leary JJ, Finn SP. Molecular testing in oncology: problems, pitfalls and progress. Lung Cancer. 2014;83(3):309-15.

9. Xu CW, Cai XY, Shao Y, Li Y, Shi MW, Zhang LY, et al. A case of lung adenocarcinoma with a concurrent EGFR mutation and ALK rearrangement: a case report and literature review. Mol Med Rep. 2015;12(3):4370-5.

10. Yang JJ, Zhang XC, Su J, Xu CR, Zhou Q, Tian HX, et al. Lung cancers with concomitant EGFR mutations and ALK rearrangements: diverse responses to EGFR-TKI and crizotinib in relation to diverse receptors phosphorylation. Clin Cancer Res. 2014;20(5):1383-92.

11. Yang JJ, Chen HJ, Yan HH, Zhang XC, Zhou Q, Su J, et al. Clinical modes of EGFR tyrosine kinase inhibitor failure and subsequent management in advanced non-small cell lung cancer. Lung Cancer. 2013;79(1):33-9.

12. Soria JC, Wu YL, Nakagawa K, Kim SW, Yang JJ, Ahn MJ, et al. Gefitinib plus chemotherapy versus placebo plus chemotherapy in EGFR-mutationpositive non-small-cell lung cancer after progression on first-line gefitinib (IMPRESS): a phase 3 randomised trial. Lancet Oncol. 2015;16(8):990-8.

13. Murtaza M, Dawson SJ, Tsui DW, Gale D, Forshew T, Piskorz AM, et al. Noninvasive analysis of acquired resistance to cancer therapy by sequencing of plasma DNA. Nature. 2013;497(7447):108-12.

14. Zhao N, Zheng SY, Yang JJ, Zhang XC, Xie Z, Xie B, et al. Lung adenocarcinoma harboring concomitant EGFR mutation and EML4-ALK fusion that benefits from three kinds of tyrosine kinase inhibitors: a case report and literature review. Clin Lung Cancer. 2015;16(2):e5-9.

15. Alì G, Chella A, Lupi C, Proietti A, Niccoli C, Boldrini L, et al. Response to erlotinib in a patient with lung adenocarcinoma harbouring the EML4-ALK translocation: a case report. Oncol Lett. 2015;9(4):1537-40.

16. Chen LC, Neubauer A, Kurisu W, Waldman FM, Ljung BM, Goodson W 3rd, et al. Loss of heterozygosity on the short arm of chromosome 17 is associated with high proliferative capacity and DNA aneuploidy in primary human breast cancer. Proc Natl Acad Sci U S A. 1991;88(9):3847-51. 